\title{
A NEW PROPOSAL FOR THE CLINICAL-FUNCTIONAL CATEGORIZATION OF THE ELDERLY: VISUAL SCALE OF FRAILTY (VS-FRAILTY)
}

\author{
E. Nunes de Moraes ${ }^{1,2}$, F.M. Lanna ${ }^{2}$, R.R. Santos ${ }^{1,2}$, M.A.C. Bicalho ${ }^{1,2}$, C.J. Machado ${ }^{1,3}$, D.E. Romero ${ }^{4}$
}

\begin{abstract}
Ageing is closely associated with increasing frailty, and it continues to be defined in various different ways, complicating its practical application in the clinical context. Frailty is commonly used to predict the risk of functional decline, institutionalization or even death. Current models of frailty display significant limitations, because they are unable to encompass all the chronic conditions indicating adverse outcomes. In this article, we propose the Visual Scale of Frailty (VS-Frailty), based on the overall functionality and clinical complexity of elderly individuals. This scale emphasizes the importance of independence in basic, instrumental and advanced Activities of Daily Living (ADL), as well as taking into account the main causes of the functional decline of the elderly, such as sarcopenia syndrome, mild cognitive impairment and multiple comorbidities. The scale places the elderly into 10 categories ranging from completely independent to fully dependent. The scale provides the following advantages over other scales currently used: scope, ease of application, visual clarity, longitudinal monitoring, planning of health services, and the definition of therapeutic goals and prioritization of care. The use of VS-Frailty permits the individualization of the overall diagnosis, thus facilitating continuity of care in a systematized manner, ensuring comprehensive attention to the health of elderly individuals.
\end{abstract}

Key words: Frailty, disability, classification of the elderly.

\section{Introduction and Motivation}

Aging has profound consequences for organization of healthcare systems due to higher incidences of chronic diseases and functional disabilities in elderly populations. However, while diseases may be more common amongst old people, they are not always linked to functional dependency. Ageing, therefore, is not synonymous of disability and dependence, but is related to greater vulnerability.

Aging alone can lead to chronic diseases, but not to functional dependency. Individuals with similar clinical diagnoses may display entirely different functional capacities, and this striking heterogeneity increases with ageing (1). As a result, various authors have asserted the need for a more sophisticated classification of the health status of the elderly $(2,3)$. We believe that

\footnotetext{
1. Department of Clinical Medicine at the Federal University of Minas Gerais, Brazil; 2. Center for Geriatrics and Gerontology UFMG, Brazil; 3. Department of Preventive and Social Medicine at the Federal University of Minas; 4. Oswaldo Cruz Foundation, Rio de Janeiro, Brasil.

Corresponding Author: Edgar Nunes de Moraes, Professor of the Department of Clinical Medicine at the Federal University of Minas Gerais, Brazil. Director of the Center for Geriatrics and Gerontology UFMG, 117, Alameda Álvaro Celso. Zip Code: 30.150-260, Belo Horizonte. Minas Gerais, Phone: (55) 31 3226-2386, Fax: (55) 31 3226-2386, Email: edgarnmoraes@gmail.com
}

functional capacity should be at the core of any health status classification. Boundaries between the concepts of health, disease, dependency and disability should be also clarified. In this way, health may be defined as a measure of individual's ability to achieve their aspirations and satisfy their needs, rather than simply as the absence of disease (4).

Well-being and functionality are complementary. Together, they represent the presence of autonomy (individual decision-making power and control over one's actions, establishing and following one's own convictions) and independence (the ability to achieve something without external assistance), allowing individuals to look after themselves and their lives. It is important to bear in mind while autonomy and independence are closely related, they are distinct concepts. Physically dependent individuals may still be capable of making their own decisions about daily activities, just as others may be physically able to perform these activities, whilst being incapable of making decisions about the nature of their involvement. According to the International Classification of Functioning, functional decline is defined as a loss of autonomy and/or independence, as it restricts many aspects of life, including social functioning5. Independence and autonomy are thus closely related to 
complete and harmonious functioning of the following capacities:

- Cognition: the mental capacity to understand and adequately resolve everyday problems;

- Mood/Behaviour: the motivation required to carry out activities and/or participate socially. This includes individual behaviour, which is affected by other mental functions such as sensory perception, thought and conscience.

- Mobility: the individual capacity to move and manipulate one's environment. This depends on four functional subsystems: aerobic and muscular capacity (mass and function), reach / prehension / grip (upper limbs) and gait / posture / transferring (lower limbs). Control of urinary and fecal sphincters is also considered a pre-requisite for mobility, as incontinence may reduce mobility and restrict the social participation of the individual.

- Communication: the ability to establish a productive relationship with one's environment, exchange information and express desires, ideas and feelings. This depends on three functional subsystems: vision, hearing and orofacial motor function. The latter refers to the voice, speech and the ability to chew and swallow.

As Moraes (4) writes, the impairment of these systems - manifested in cognitive disability, postural instability, incontinence, immobility and the inability to effectively communicate. These syndromes are known as "geriatric syndromes". Besides that, presence of chronic medical conditions increases the complexity of clinical management, thus implying increased risk of iatrogenesis. Functional decline and frailty are closely related. The term 'frailty' is commonly used to express the degree of vulnerability of the elderly individual to adverse outcomes like functional dependency. It is also used to refer to other levels of vulnerability, such as the risk of institutionalization, or even death. The fact that the term has been defined in several different ways complicates its application in the clinical context $(6,7)$.

\section{Literature review and multidimensional frailty}

Fried and colleagues $(8,9)$ and Rockwood and colleagues $(10,11)$ are among the authors who have examined this concept in detail. In 2001, Fried proposed the term 'frailty' to represent a geriatric syndrome, of multifactorial nature, characterized by a reduction in energy levels and resistance to stressors, resulting in the cumulative decline of physiological systems. This concept was operationalized in the "phenotype of frailty', validated by the Cardiovascular Health Study. It is characterized by three or more of the following criteria: involuntary weight loss (5 $\mathrm{kg}$ in the last year), self-declared exhaustion (according to the Center for Epidemiologic Studies Depression Scale/CES-D), weakness (reduction in muscular strength assessed by grip strength), low level of physical activity (verified by the Minnesota Leisure Time Activities Questionnaire and reduced walking speed (over a timed walk of $4.5 \mathrm{~m}$ ). The presence of three or more of these features defines the individual as 'frail', while the presence of two defines them as 'pre-frail'. Individuals who do not display any of these criteria are considered robust. This classification excludes individuals suffering from comorbidity and functional dependency and there is a high overlap between the three groups.

In 2005, Rockwood proposed a broader model of frailty, based on the presence of cumulative clinical deficits. This was expressed by the Frailty Index (FI), the result of the application of the Comprehensive Geriatric Assessment. The FI is a weighted measurement, derived from individual accumulation of 30 to 70 clinical problems, including specific diseases (congestive cardiac failure, arterial hypertension, strokes, myocardial infarction, Parkinson's disease and cancer, among others), symptoms (shaking, bradykinesia, cephalalgia, syncope, among others), functional loss (such as dependence in daily activities, memory loss, problems with mood, changes in gait and balance, falls, immobility and urinary and fecal incontinence) and abnormal laboratory tests. Yet, one criticism that can be made is that the FI is comprehensive, but very broad.

Both Fried's and Rockwood's models of frailty have important limitations. Fried's model is incapable of encompassing all the chronic medical conditions associated with greater risk of functional decline, hospitalization and death. It considers only mobility, in particular, aerobic and muscular capacity, underestimating the importance of other functional capacities such as cognition (12). Moreover, its application requires the use of handgrip equipment, which is not widely available in primary care. Rockwood's Frailty Index uses a mathematical model based on the accumulation of problems, which are not clearly defined or standardized, and requires the application of a comprehensive geriatric assessment. This assessment brings its own problems, as there is no consensus regarding its structure, and it varies widely in its application. The current challenge, therefore, is to operationalize the concept of frailty, facilitating its recognition and the implementation of actions capable of maximizing individual independence and autonomy and preventing adverse outcomes (13), thereby making the term useful for those considering the prevention of functional disability in the elderly.

Aligned with the idea of understanding frailty in its broader meaning, we propose the concept of multidimensional frailty. It is defined as the reduction of the homeostatic reserve and/or the ability to adapt to biopsychosocial problems, and consequently, greater susceptibility to functional decline. In this multidimensional model, the conditions associated 
with adverse outcomes can be placed in two categories: clinical-functional and socio-familial. The concept of health, for the elderly individual, should therefore consider information about both these aspects, according to WHO definition of health as a state of complete physical, mental and social well-being and not merely the absence of disease or infirmity.

Social conditions, including socioeconomic status, the presence of social support, social engagement and the feeling of self-sufficiency, exert a decisive influence on health, and indeed on an individual's survival, particularly amongst the elderly $(14,15)$. For this reason, the socio-familial category considers the importance of factors such as gender, race, level of education, marital status, familial arrangements, recent widowhood, if they are themselves a carer, extent of social participation and support, access to health services and employment situation. Some of these considerations are qualitative and difficult to measure, which limits their use in structured scales.

The variables or factors considered in the clinicalfunctional category are better structured, though a lack of consensus persists. It takes into account biological, physical, cognitive and psychological factors responsible for functional decline in the elderly. Ageing increases the risk of health problems (senility) related to diseases and external causes (such as traumas and accidents), which are usually manifest in the form of multiple comorbidities (polypathology, polypharmacy and recent hospitalization) and represent the principal cause of disability in the elderly. On the other hand, normal ageing (senescence) may also be associated with the decline of homeostatic reserves and increased susceptibility to problems, principally through immunological activation, which generates a chronic proinflammatory state. This in turn permits the development of sarcopenia syndrome, manifested in the reduction of aerobic and muscular capacities, which are considered the principal elements in the phenotype of Frailty (8). Senility and senescence may both therefore lead to functional dependence. Disabilities further compromise homeostatic reserves, producing a vicious cycle, leading to progression of the disabilities, hospitalization and even death. In this multidimensional model, we take into account Fried's and colleagues' and Rockwood's and colleagues' models, as they are not considered mutually exclusive. On the contrary, they are complementary or synergic, and may both be present in the same individual (Figure 1). The model thus preserves the differences between comorbidities, disabilities and the 'phenotype of Frailty'. It considers all the components of frailty in the elderly, understood as the presence of disabilities and / or chronic conditions associated with functional decline, institutionalization and/or death.

Functional decline is therefore the main target of geriatric-gerontological assessment and therapeutic interventions. In this model, functional decline is posited as established and imminent. Imminent functional decline is understood as the presence of chronic conditions indicative of functional dependency, such as sarcopenia and the presence of multiple comorbidities (polypathology, polypharmacy and recent hospitalization).

It is worth re-emphasizing the distinction between sarcopenia as a process and sarcopenia as a syndrome. The process of sarcopenia, understood as the reduction of muscular mass, is the result of neuroendocrine alterations which are responsible for changes to the composition of the ageing body. Such changes include reductions in muscular mass, bone mass and body water content, as well as an increase in fat. The syndrome of sarcopenia, on the other hand, is characterized by the presence of changes in muscular mass and function (strength and muscular performance), that is strictly related to the 'phenotype of frailty'. In the proposed model, the criteria for the diagnosis of sarcopenia include the presence of three of more features from the phenotype of frailty (weight loss, exhaustion, grip strength, low level of physical activity and a reduction in walking pace), as well as quantitative measurements of muscular mass, associated with measurements of muscular function, such as grip force and walking speed.

A reduction in gait speed is one of the principal determinants of sarcopenia / dynapenia, defined as a gait speed equal to or less than $0.8 \mathrm{~m} / \mathrm{s}$. Another strong indication of sarcopenia is a calf circumference equal to or less than $31 \mathrm{~cm}$. Other indications of sarcopenia are significant non-intentional weight loss, or low body mass index (BMI) (less than $22 \mathrm{~kg} / \mathrm{m} 2)$ (16-18). Recently, some authors have suggested that the reduction in neuropsychological reserve is another indication of adverse outcomes in the cognitive area. 'Cognitive Frailty' is defined as a heterogeneous clinical syndrome characterized by the presence of physical Frailty ('phenotype of Frailty') associated with cognitive decline involving mild cognitive impairment (CDR 0.5) (19).

The presence of multiple medical conditions is related to increasing use of healthcare systems and higher risk of iatrogenesis, disabilities, institutionalization and even death (20). Thus, multiple medical conditions contribute to imminent functional decline. However, the definition of multimorbidity, and its operationalization, have yet to be clearly established. Multimorbidity can be defined as a combination of acute and/or chronic medical conditions, associated with a range of biopsychosocial risk factors, such as polypharmacy, lifestyle habits, and excessive use of healthcare systems, among others (21). In the model proposed here, we define multimorbidity as the presence of one or more of three clinical conditions: polypathology (simultaneous presence of five or more chronic medical conditions, attacking different physiological systems); polypharmacy (regular and systemic use of five or more medications per day for different chronic conditions); or recent hospitalization (in the last six months). 
In addition to the presence of multiple comorbidities, evidence of sarcopenia syndrome and mild cognitive impairment, we include the presence of established functional decline as a determinant of Frailty. As such, functional dependency may be considered both a cause, and at the same time, an effect of Frailty $(22,23)$.

The overall functionality of the individual has always been fundamental for any classification of the elderly. In 1943, Marjory Warren, considered the 'mother of geriatrics' (24), proposed a classification based on the presence of various types of disability, such as postural instability, immobility, urinary incontinence and cognitive disability. This pioneering classification of elderly patients at an infirmary enabled the planning of the human and material resources necessary to implement specific actions for each group of elderly individuals. In 1963, Katz developed an index capable of categorizing individuals according to the level of dependence in Activities of Daily Living (ADL) related to self-care (25). The Katz index made a clear distinction between independent and dependent individuals in ADL, and included a hierarchy for the dependent individuals. Elderly individuals may therefore be categorized as:

- Independent: capable of carrying out basic daily activities in an independent manner;

- Semi-dependent: displays difficulty in carrying out one of the functions influenced by culture and learning (bathing and/or getting dressed and/or using the toilet);

- Incomplete dependent: displays impairment in one of the simple vegetative functions (transferring and/or continence), as well as requiring assistance in bathing, getting dressed and using the toilet. The isolated presence of urinary incontinence should not be taken into account, as it is a function rather than an activity;

- Complete dependent: displays impairment in all functions influenced by culture and learning, as well as in simple vegetative functions, including the ability to eat without assistance. This is the maximum degree of functional dependence.

In 1969, Lawton and Brody (26) confirmed the importance of assessing the functionality of the individual in their basic daily tasks, but they also proposed the assessment of more complex abilities, denominated instrumental ADL, related to selfmaintenance. Elderly individuals may therefore be independent, partly dependent or completely dependent in instrumental ADL. Usually, functional decline follows a hierarchy, beginning with the more complex (advanced and instrumental) ADL, until the impairment of activities related to self-care (basic ADL). Moreover, it may be a consequence of impairment in isolated functions or connected functional areas. Clinico-functional classification of the elderly individual depends directly upon this functional classification. These scales (Katz's and Lawton-Brody's scales) are vital for the objective assessment of the elderly individual, and for the creation, implementation and evaluation of a care plan.

Figure 1

Ageing and Frailty

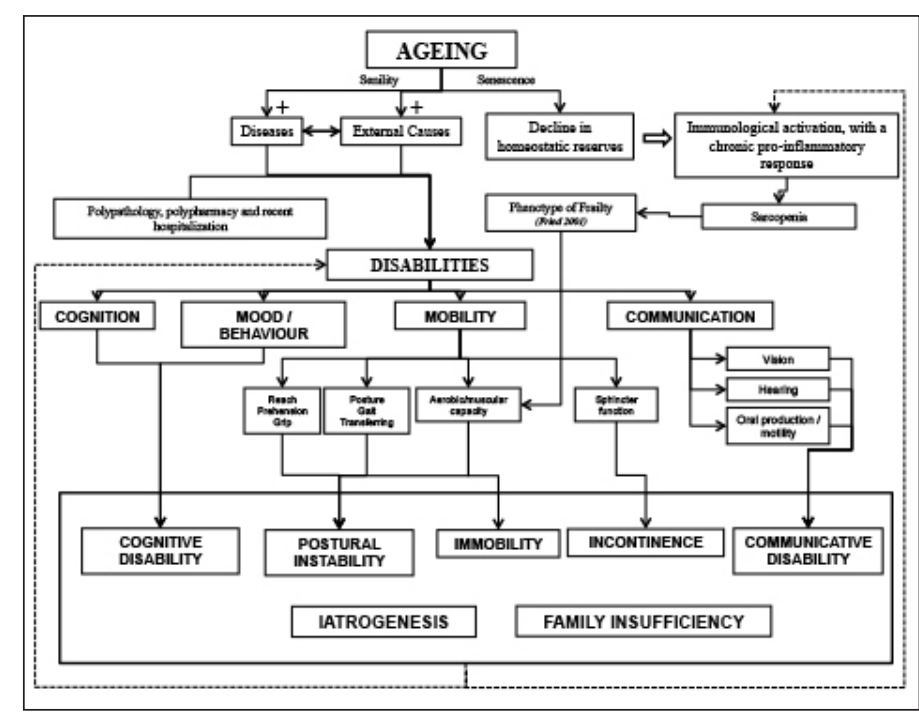

\section{The VS-Frailty proposal}

While ageing is closely linked to the frailty there is no direct cause and effect relation between the two. The two phenomena do not follow the same pattern of evolution, and elderly individuals of the same age may display highly heterogeneous conditions (27). Thus, age alone is not a good indication of Frailty.

To express more clearly the various sub-groups of elderly individuals, we propose the use of the Visual Scale of Frailty (VS-Frailty), based on their functionality and clinical complexity. Visual analogic scales have been used to assess a range of issues related to human health, particularly those of a multidimensional nature and those which display a dynamic character over time, such as pain, mood and dyspnoea, among others 28 . This permits continuous measurement within an established set of values, in a linear and proportional manner, taking into account both objective and subjective factors. The VS-Frailty thus encompasses extremes, from the maximum and minimum degree of vitality (homeostatic reserve) and frailty (vulnerability). These extremes are linked by a straight line, continuous and declining, upon which the health of the elderly individual may be marked. Ten clinical-functional categories were defined, which vary continuously within the pre-established range of values. Elderly individuals may therefore be categorized as illustrated in Figure 2.

ROBUST: Elderly individuals who display good homeostatic reserve, being therefore capable of managing their lives in an independent and autonomous manner. They do not display any functional disability or any chronic medical condition associated with 27 
the maintenance of the individual's autonomy and independence, through measures which promote health, as well as primary and secondary preventive measures and appropriate clinical management of diseases according to their respective established guidelines. This group is divided into three sub-categories:

- Category 1: Elderly individuals who display the maximum degree of vitality. They retain independence in all advanced, instrumental and basic ADL, as well as being free of illness and other risk factors apart from their age. They do not display any medical conditions indicative of adverse outcomes.

- Category 2: Elderly individuals independent in all ADL, but who display medical conditions of low clinical complexity, such as arterial hypertension (without complications) and/or the presence of risk factors such as tobacco smoking, dyslipidaemia and osteopenia, among others.

- Category 3: Elderly individuals independent in all ADL, but who display well-established chronic degenerative diseases of higher clinical complexity, such as complicated arterial hypertension, diabetes mellitus, a history of transient ischemic attacks, strokes (without further consequences), chronic renal disorder, heart failure, chronic obstructive pulmonary disease, osteoarthritis; coronary artery disease, with or without acute myocardial infarction; peripheral artery disease, cancer, osteoporosis and atrial fibrillation, among others. In these individuals, the presence of these conditions is not associated with functional limitation, and in general, they appear in isolation. Individuals displaying one or two criteria of the 'phenotype of Frailty' also fall into this category.

AT RISK OF FRAILTY: Elderly individuals capable of managing their life in an independent and autonomous manner, who remain in a dynamic state between senescence and senility, resulting in the presence of certain functional limitations (imminent functional decline), but without functional dependency. They display one or more of the chronic medical conditions indicative of adverse outcomes, such as evidence of sarcopenia syndrome, mild cognitive impairment and/or the presence of multiple comorbidities. The aim of treatment must be to prevent functional decline through the correct handling or correct approach to managing polypathology and polypharmacy, with an emphasis on the following, in order of importance: suspension of inappropriate medication, definition of personalized therapeutic goals, prioritization of care, diagnosis and treatment of underdiagnosed and/or undertreated medical conditions, rehabilitation, primary and secondary prevention. This group corresponds to categories four and five of the VS-Frailty:

- Category 4: These are elderly individuals who display symptoms of sarcopenia syndrome, mild cognitive impairment (MCI) and/or multiple comorbidities (polypathology, polypharmacy or recent hospitalization), but who remain independent in all ADL, including advanced ADL, defined as activities related to social integration, productive, recreational and/or social activities.

- Category 5: These are elderly individuals who display symptoms of sarcopenia syndrome, mild cognitive impairment and/or multiple comorbidities, and who also have begun to display functional decline in advanced ADL. However, they remain independent in instrumental and basic ADL.

\section{Figure 2}

Visual Analogue Scale of Frailty (VAS-Frailty)

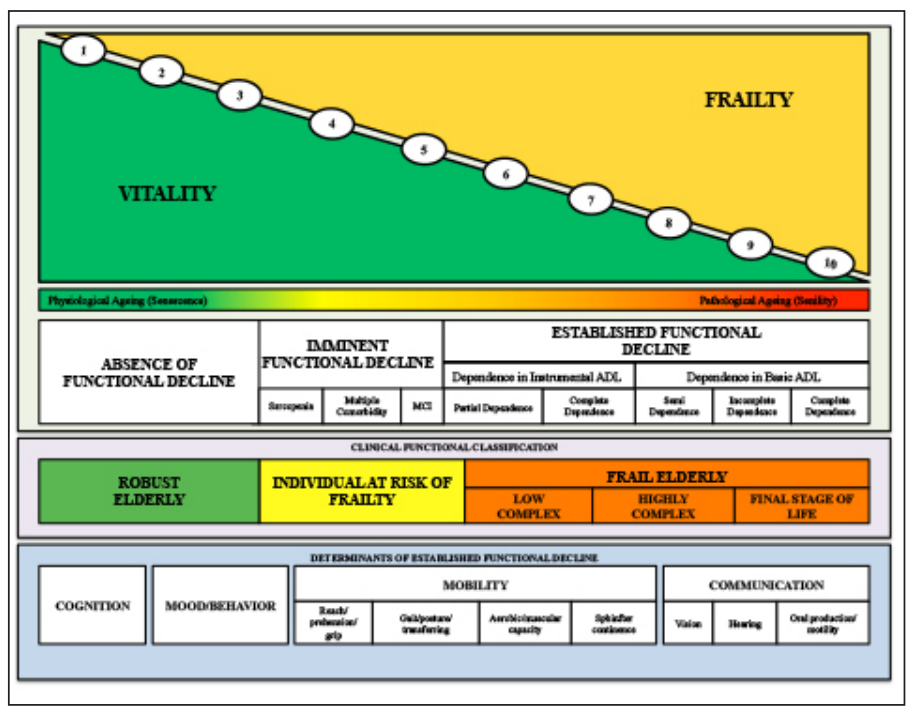

From Category 6 on, classification is essentially based on the presence of established functional decline, which is the consequence of major geriatric syndromes such as cognitive disability, postural instability, incontinence and communicative disability. These patients are also associated with iatrogenesis and family insufficiency, and are likely to suffer from other chronic medical conditions.

FRAIL: Elderly individuals suffering from established functional decline, who are incapable of managing their own lives, as a result of single or multiple disabilities. The aim of treatment should be the recovery of the individual's autonomy and independence. This group corresponds to the categories six, seven, eight, nine and ten of the VS-Frailty:

- Category 6: Individuals who display partial functional decline in the instrumental activities of daily living, but who remain independent in basic ADL.

- Category 7: Individuals who display functional decline in all the instrumental activities of daily living, but who remain independent in basic ADL.

- Category 8: Individuals who display total functional decline in instrumental ADL, as well as semidependence in basic ADL: impairment of one of the functions influenced by culture and learning (bathing, getting dressed and/or using the toilet). 
- Category 9: Individuals who display total dependence in instrumental ADL, alongside partial dependency in basic ADL: impairment of one of the simple vegetative functions (movement and/or continence), as well as requiring assistance to bathe, get dressed and use the toilet. The isolated presence of urinary incontinence should not be taken into account, as it is a function and not an activity.

- Category 10: Individuals who display the maximum degree of Frailty, and therefore the highest level of functional dependence. They require assistance even to eat. On Katz's index, they are classified at Stage G (25).

Definition of the clinical-functional category should consider the best current performance of ADL. So, if the individual is capable of various instrumental ADL, they should be placed in category 7 , even if they are unable to carry out a basic ADL. As well as functional heterogeneity, frail individuals also display differences regarding clinical complexity and the need for specialized geriatric-gerontological assistance. In this sense, frail individuals may also be categorized according to the following definitions:

o Low Complex Frail Individual: Displays established functional decline, with low potential for clinicalfunctional reversibility. With these individuals, intense geriatric-gerontological assistance is not required and the aim of treatment should be the prevention of functional deterioration.

o Highly Complex Frail Individual: Displays functional dependency in ADL, together with medical conditions that are difficult to manage as a result of doubts regarding diagnosis or therapy. The term 'Highly Complex' suggests high potential for clinico-functional reversibility or quality of life. These individuals benefit most from intensive monitoring by teams of geriatricgerontological specialists.

o Frail Individual in Final Stage of Life: Displays a high degree of functional dependency and life expectancy is estimated at less than six months. Despite the existence of a clear link between higher levels of functional decline and higher mortality rates, some individuals may be able to function relatively well despite suffering from diseases that present a high risk of mortality, such as certain types of neoplasia (terminally ill). The aim of treatment should be palliative care, based on the comfort of the patient and their family members.

The scale provides the following advantages over the scales already discussed and currently used:

- Scope: the scale is based on the multidimensionality of factors that determine geriatric health $(29,30)$, without minimizing the importance of diseases or physical changes, including the frailty indicators as described by Fried and colleagues (9), as well as diseases, polypathology and polypharmacy;

- Ease of application: it does not require any sophisticated or hard-to-obtain tools, and may be applied in any environment of geriatric care, such as outpatients' clinics, patients' homes, care homes, day centres and hospitals. It unites objective and subjective aspects in its assessment of elderly individuals.

- Visual clarity: the scale is visual and it incorporates fundamental concepts for the understanding of the ageing process, such as senescence, senility, vitality, frailty, functional decline and ADL. The categories 1 to 10 take into account the heterogeneity of elderly individuals of the same age group;

- Longitudinal monitoring: permits longitudinal monitoring of the health of the elderly individual, facilitating the visualization of the results of geriatricgerontological treatment;

- Planning of health services: the scale establishes objective criteria for geriatric-gerontological care;

- Definition of therapeutic goals and prioritization of care: the clinico-functional categorization permits the definition of personal and specific objectives for each patient. With robust individuals, therapeutic goals will be similar to clinical guidelines regarding diseases. By contrast, with highly dependent individuals, or with individuals reaching the end of their life, therapeutic goals must prioritize comfort, as functional loss is critical, and classical preventive strategies should be revised.

The use of VS-Frailty makes possible the individualization of the overall diagnosis of the individual, thus facilitating continuity of care in a systematized manner, ensuring comprehensive attention to the health of the elderly individual.

\section{Conflict of interest: No potential conflicts of interest were disclosed.}

Author Contributions: Edgar N. Moraes: study concept and design, preparation of paper. Flávia M. Lanna: study concept and design, preparation of paper. Rodrigo R. Santos: study concept and design, preparation of paper. Maria A.C Bicalho: study concept and design, preparation of paper. Carla J. Machado: study concept and design, preparation of paper. Dália E. Romero: study concept and design

\section{References}

1. Veras RP. Gerenciamento de doença crônica: equívoco para o grupo etário dos idosos. Rev Saúde Pública 2012; 46: 929-34.

2. Ramos LR. Fatores determinantes do envelhecimento saudável em idosos residentes em centro urbano: Projeto Epidoso, São Paulo. Cad. Saúde Pública 2003; 19: 793-798.

3. Cesari M, Gambassi G, Van Kan GA, Vellas B. The frailty phenotype and the frailty index: different instruments for different purposes. Age and Ageing 2014; 43: 10-12.

4. Moraes EN. Atenção à Saúde do Idoso: Aspectos Conceituais. Brasília: Organização Pan-Americana da Saúde, 2012.

5. Organização Mundial de Saúde / Organização Panamericana de Saúde. CIF - Classificação Internacional de Funcionalidade, Incapacidade e Saúde: Universidade de São Paulo; 2003.

6. Rodrígues-Mañas LR, Féart C, Mann G et al. Searching for an Operational Definition of Frailty: A Delphi Method Consensus Statement. The Frailty Operative Definition-Consensus Conference Project. J Gerontol A Biol Sci Med Sci 2013; 68: 62-67.

7. Gordon AL, Masud T, Gladman JRF. Now that we have a definition for 
physical frailty, what shape should frailty medicine take? Age and Ageing 2014; 32: 8-9.

8. Fried LP et al. Frailty in older adults: evidence for a phenotype. J. Gerontol. A. Biol. Sci. Med. Sci. 2001; 56: M146-156.

9. Fried LP et al. Untangling the Concept of Disability, Frailty and Comorbidity: Implications for Improved Targeting and Care. J. Gerontol. A. Biol. Sci. Med. Sci. 2004; 59: 255-263.

10. Rockwood $\mathrm{K}$ et al. A global clinical measure of fitness and frailty in elderly people. CMAJ 2005; 173: 489-495.

11. Rockwood K, Mitnitski A. Frailty defined by deficit accumulation and geriatric medicine defined by frailty. Clin Geriatr Med 2011; 27: 17-26.

12. Martin FC, Brighton P. Frailty: different tools for differents purposes? Age and Ageing 2008; 37: 129-131.

13. Clegg A et al. Frailty in elderly people. Lancet 2013; 8: 1-11.

14. Andrew MK et al. The impact of social vulnerability on the survival of the fittest older adults. Age Ageing 2012; 41: 161-165.

15. Gobbens RJJ et al. Towards an integral conceptual model of frailty. J Nutr Health Aging 2010; 14: 175-81.

16. Sayer AA et al. New horizons in the pathogenesis, diagnosis and management of sarcopenia. Age and ageing 2013; 42:145-150.

17. Cruz-Jenkoft AJ et al. Sarcopenia: European consensus on definition and diagnosis. Age and Ageing 2010; 39: 412-423.

18. Fielding RA et al. Sarcopenia: An Undiagnosed Condition in Older Adults. Current Consensus Definition: Prevalence, Etiology, and Consequences. International Working Group on Sarcopenia. J Am Med Dir Assoc 2011; 12: 249-256.

19. Kelaiditi E, Cesari M, Canevelli M. et al. Cognitive Frailty: Rational and Definition from an (I.A.N.A./I.A.G.G.) International Consensus Group. The Journal of Nutrition, Health \& Aging 2013; 17: 726-734.

20. American Geriatrics Society Expert Panel on The Care of Older Adults With
Multimorbidity. Patient-Centered Care for Older Adults with Multiple Chronic ,Conditions: A Stepwise Approach from the American Geriatrics Society 2012.

21. Reste JYL, Nabbe P, Manceau B. et al. The European General Practice Research Network Presents a Comprehensive Definition of Multimorbidity in Family Medicine and Long Term Care, Following a Systematic Review of Relevant Literature. JAMDA 2013; 14: 319-325.

22. Landi F Liperoti R, Russ et al. A Disability, more than multimorbidity, was predictive of mortality among older persons aged 80 years and older Journal of Clinical Epidemiology 2010; 63: 752-759.

23. Sternberg AS, Schwartz AW, Karunananthan S. et al. The identification of Frailty: A Systematic Literature Review. JAGS 2011; 59: 2129-2138.

24. Warren MW. Care of Chronic Sick: a case for treating chronic sick in blocks in a general hospital. British Medical Journal 1943; ii:822-823.

25. Katz S. Ford AB, Moskowitz RW et at. Studies of Illness in the Aged. The Index of ADL: A Standardized Measure of Biological and Psychosocial Function. JAMA, 1963; 185:94-99.

26. Lawton MP, Brody EM. Assessment of Older People: Self-Maintaning and Instrumental Activities of Daily Living. Gerontologist 1969; 9: 179-185.

27. Lacas A; Rockwood K. Frailty in primary care: a review of its conceptualization and implications for practice. BMC Med 2012; 10, n. 4, 11

28. Wewers ME, Lowe NK. A Critical Review of Visual Analogue Scales in the Measurement of Clinical Phenomena. Research in Nursing \& Healty 1990; 13: 227-236.

29. Morley JE, Vellas B, Abellan VKG et al. Frailty Consensus: A Call to Action. JAMDA 2013; 14: 393-397.

30. Berrut G., Andrieu S., Carvalho A.I. et al. Promoting access to innovation for frail old persons. The Journal of Nutrition, Health \&Aging 2013;7:1-6. 